\title{
Latest measurements of neutrino oscillation parameters with the T2K experiments and its future plans
}

\author{
Stephen Dolan for the T2K collaboration ${ }^{a, *}$ \\ ${ }^{a} C E R N$, \\ European Organization for Nuclear Research, Geneva, Switzerland \\ E-mail: stephen.joseph.dolan@cern.ch
}

Tokai-to-Kamioka (T2K) is a long baseline neutrino experiment which uses the proton beam at the Japan Particle Accelerator Research Centre (JPARC) to produce a beam of muon neutrinos and antineutrinos. T2K determines neutrino oscillation parameters of interest by comparing the measured neutrino rate and spectrum at a near detector complex, located at JPARC, and at SuperKamiokande, a water-Cherenkov detector, located $295 \mathrm{~km}$ away. T2K is currently undergoing major improvements: the JPARC neutrino beam is increasing in power, the Super-Kamiokande detector has been loaded with $0.02 \%$ of Gadolinium, enabling enhanced neutron tagging; and a significant upgrade of the ND280 near detector is scheduled for installation in 2022/23. These proceedings summarise T2K's latest measurements of neutrino oscillations and detail some of the planned improvements, with particular focus on the upcoming near detector upgrade.

*** The European Physical Society Conference on High Energy Physics (EPS-HEP2021), ***

*** 26-30 July $2021 * * *$

*** Online conference, jointly organized by Universität Hamburg and the research center DESY ***

${ }^{*}$ Speaker 


\section{Neutrino oscillations measurements with the T2K experiment}

The T2K experiment is well equipped to make precise measurements of neutrino oscillations [1, 2]. It is particularly able to constrain the $\theta_{23}, \Delta m_{32}^{2}$ and $\delta_{C P}$ parameters within the PMNS parameterisation of 3-flavour neutrino oscillations [3,4]. To achieve this, neutrinos are first produced at JPARC by impinging a beam of $\sim 30 \mathrm{GeV}$ protons into a carbon target and magnetically focusing subsequently produced mesons into a decay volume [5]. These decay into predominantly muon neutrinos (in " $v_{\mu}$ mode") or muon anti-neutrinos (in " $\bar{v}_{\mu}$ mode"), depending on whether positively or negatively charged mesons are focused. The resultant neutrino beam peak energy is $\sim 0.6 \mathrm{GeV}$ [5]. The beam is measured both immediately after production at a suite of "near" detectors (ND) and after $295 \mathrm{~km}$ at the $50 \mathrm{kT}$ Super-Kamiokande water-Cerenkov "far" detector (FD) [1]. Neutrino oscillations are then characterised by inferring muon neutrino disappearance and electron neutrino appearance energy spectra at the FD through the measurement observed muon and electron neutrino interaction event rates as a function of the reconstructed neutrino energy. However, in addition to any neutrino oscillation probability, this measured event rate is also a function of the incoming neutrino flux, the neutrino interaction cross section and the FD detector response.

Systematic uncertainties associated with neutrino cross sections and the incoming flux are constrained by fitting a parameterised model to neutrino interaction data from the primary "ND280" $\mathrm{ND}$, as illustrated in Fig. 1. The uncertainties associated with neutrino interactions are particularly challenging to evaluate and constrain, as these need to cover a wide array of poorly-understood nuclear physics associated with neutrino-nucleus interactions [6]. The model parameters and prior uncertainties are built to cover plausible variations in the T2K flux prediction [5], the input NEUT neutrino interaction simulation [7], and the ND280 detector response. The ND280 data is divided into 18 samples, each binned in the outgoing momentum and angle of observed muons produced in charged-current neutrino interactions. The subsequent flux and cross-section constraints are then propagated to the FD, where 5 samples of muon and electron neutrino interactions are fit with the same (constrained) flux and cross section model, in addition to a model for the FD detector response and the PMNS neutrino oscillation parameters themselves ${ }^{1}$. Examples of the measured FD spectra overlaid with the modelled prediction following the fit are shown in Fig. 2. The extracted constraints on neutrino oscillation parameters are given in Fig. 3. The latest T2K results weakly prefer the upper octant and are compatible with maximal mixing. There is also a weak preference for the normal mass ordering. It is found that $\delta_{C P}=-1.97_{-0.70}^{+0.97}$ and the CP-conserving values of $\delta_{C P}=0, \pi$ are excluded at more than $90 \%$ confidence level. This analysis was first presented in [8] and will be fully detailed in forthcoming publication.

With respect to T2K's latest published analysis $[2,9]$, the results shown here include $33 \%$ more $v_{\mu}$ mode data at the FD and $99 \%$ more $v_{\mu}$ mode and $116 \%$ more $\bar{v}_{\mu}$ mode data at the ND. The increased data permit improved $\bar{v}_{\mu}$ mode selections at the ND and a re-binning of all selections, thereby improving ND constraints on the systematic uncertainties. The FD also used an improved detector model. The neutrino interaction model was overhauled, including a more realistic description of nuclear effects in neutrino interactions and a more detailed set of systematic uncertainties. Finally the flux model was also updated to include improved prior constraints from the NA61/SHINE hadron production experiment at CERN [10].

\footnotetext{
${ }^{1}$ note that some parameters are not constrained at the ND and that some analyses fit the FD and ND data simultaneously.
} 

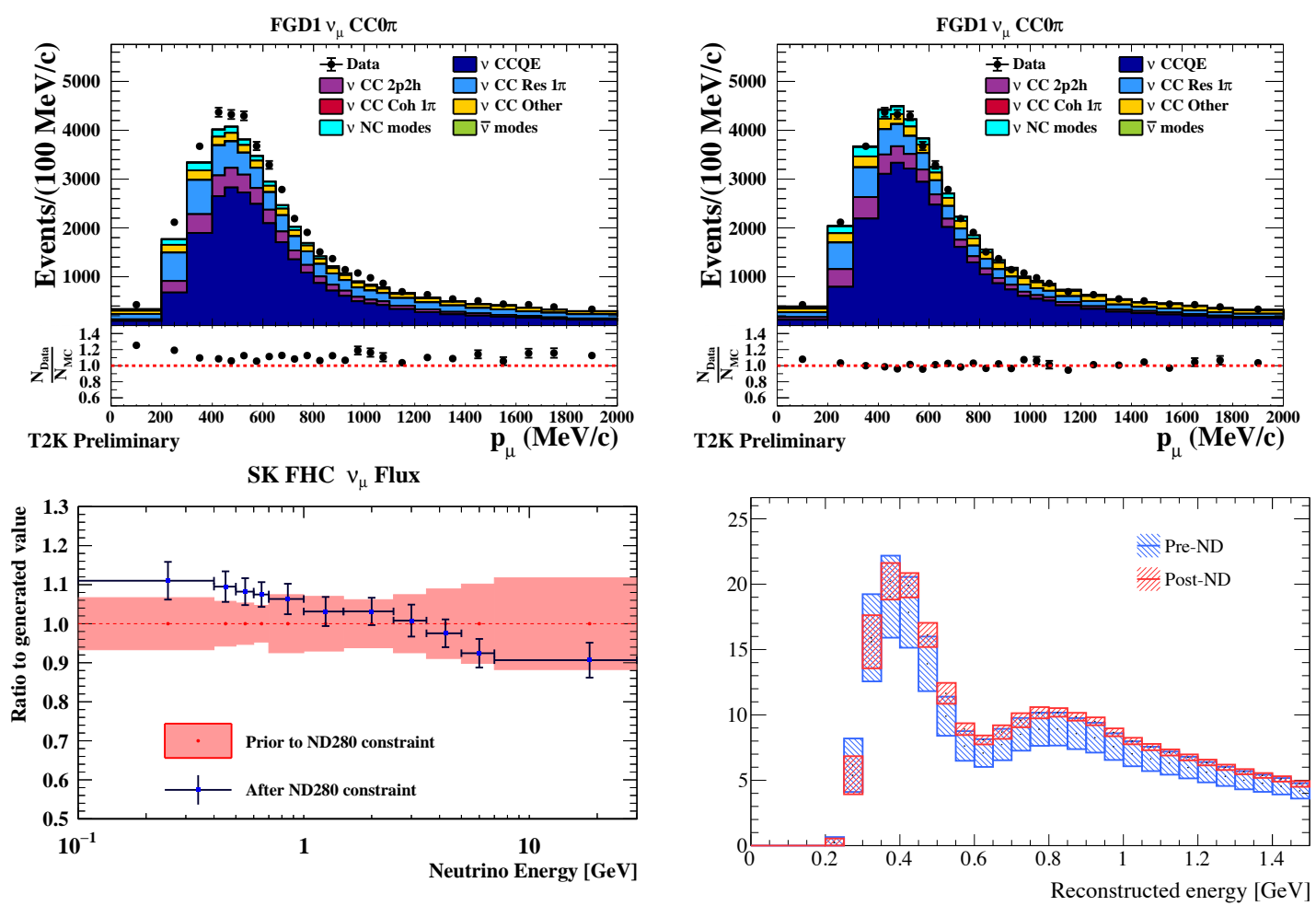

Figure 1: A summary of the ND constraints in T2K's oscillation analysis. The upper plots show an example of ND data compared to simulation before (left) and after (right) the fit to constrain the parameters. The plots show only one of 18 ND280 samples included in the fit and are a one dimensional projection of the data and simulation as a function of the measured outgoing muon momentum (the fit includes a two dimensional binning in muon momentum and angle). The lower left plot shows an example of the parameter constraints after the fit, showing a subset of the parameters controlling the neutrino flux prediction (each parameter scales the incoming flux of neutrinos of some flavour in some range of neutrino energy). The band shows the prior uncertainties and the error bars show the constraint after the fit. The lower right plot shows the predicted event rate for one of the FD event selections and the predicted uncertainties before and after the ND constraint, demonstrating the reduction in systematic uncertainty. (T2K Preliminary)
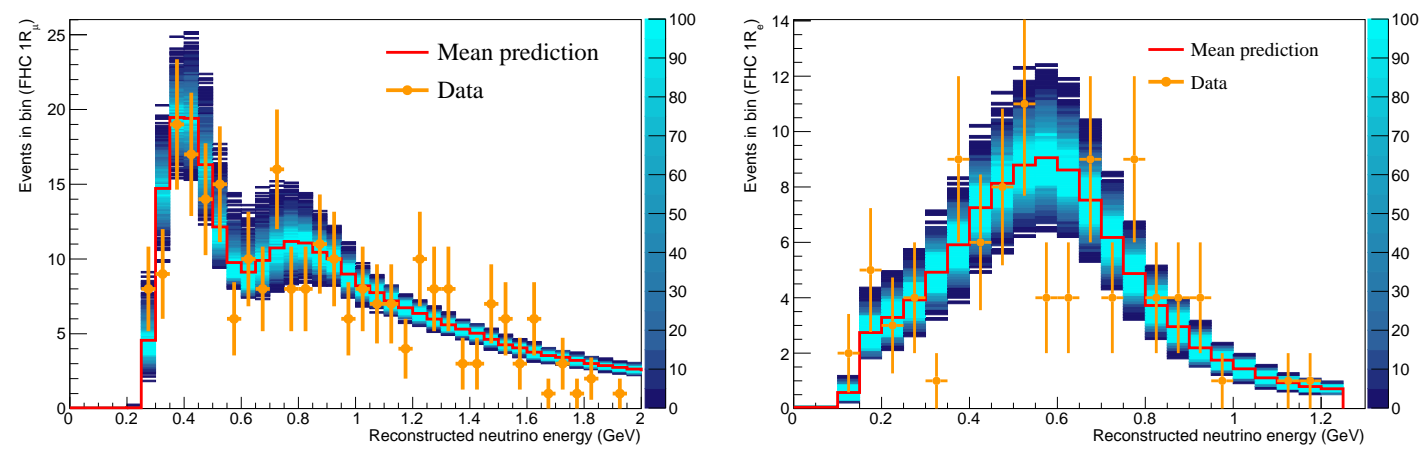

Figure 2: Two of the FD samples (one for muon neutrino disappearance on the left and one for electron neutrino appearance on the right) included in the analysis, showing the data and prediction following the FD fit to determine the oscillation parameters. The coloured band indicate the spread of the predictions covered by the post-fit simulation parameter uncertainties. (T2K Preliminary). 

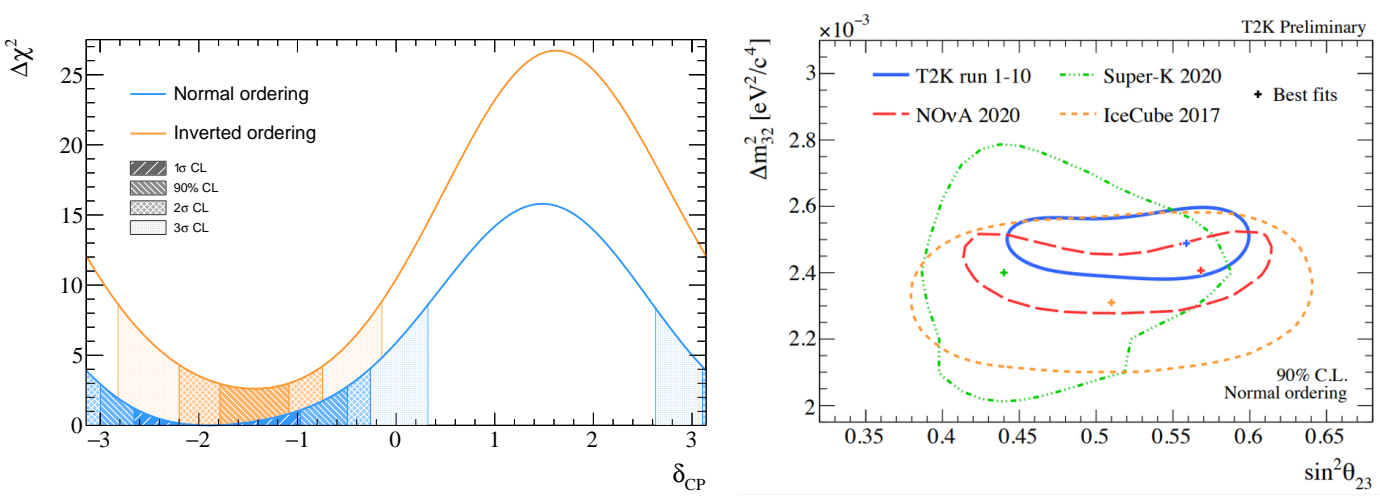

Figure 3: The constraints on the $\theta_{23}, \Delta m_{32}^{2}$ and $\delta_{C P}$ PMNS parameters following the latest T2K oscillation analysis. The left plot shows the $\Delta \chi^{2}$ distribution in $\delta_{C P}$ following the fit to data where the shaded areas indicate confidence regions of differing strengths. The results are shown for both the normal and inverted neutrino mass ordering hypotheses. The right plot shows the $90 \%$ confidence limit obtained by T2K for $\theta_{23}$ and $\Delta m_{32}^{2}$ in the case of the normal mass ordering compared to those of other experiments [11-13]. (T2K Preliminary).

\section{Upcoming improvements}

Scheduled upgrades will increase the JPARC beam power, add Gadolinium (Gd) to the FD water and significantly upgrade ND280. The JPARC upgrades will increase the beam power from its current maximum of $515 \mathrm{~kW}$ to beyond $1.3 \mathrm{MW}$, and will permit the focusing horns to run at a current of $320 \mathrm{kA}$ [14], thereby allowing T2K to further accelerate in its data taking rate. Meanwhile, Gd loading of the FD water for the SK-Gd project [15] will allow the tagging of neutrons from neutrino interactions. Sk-Gd is likely to benefit T2K oscillation analyses by allowing a better separation of neutrino and anti-neutrinos and of different neutrino interaction modes in the FD selections, thereby reducing systematic uncertainties. The ND280 upgrade is detailed below.

\subsection{The ND280 Upgrade project}

The new components of ND280 added in the upgrade are shown in Fig. 4, consisting of two horizontal "high angle" gaseous Argon TPCs (HA-TPC), a new "Super-FGD" (SFGD) and surrounding Time-of-Flight (TOF) planes [16]. The HA-TPCs [17] and TOF [18] planes will significantly extend the detector's angular acceptance whilst also improving particle identification and momentum reconstruction. The SFGD is a new fully active target for neutrino interactions made of more than 2 million $1 \mathrm{~cm}^{3}$ optically isolated scintillating cubes connected with readout fibres in three orthogonal directions [19]. This new design allows excellent hadron momentum reconstruction with low thresholds in addition to neutron detection possibilities [20]. These upgrades to ND280 are expected to be installed in 2022/23.

An overview of the expected improvement in detector performance with respect to the current ND280 are shown in Fig. 5. Overall the improved detector capabilities will allow a much more complete and robust characterisation of neutrino interactions and the incoming neutrino flux for future $\mathrm{T} 2 \mathrm{~K}$ oscillation analyses. The expected improvements in constraints on key systematic uncertainties have recently been detailed in Ref [21]. 

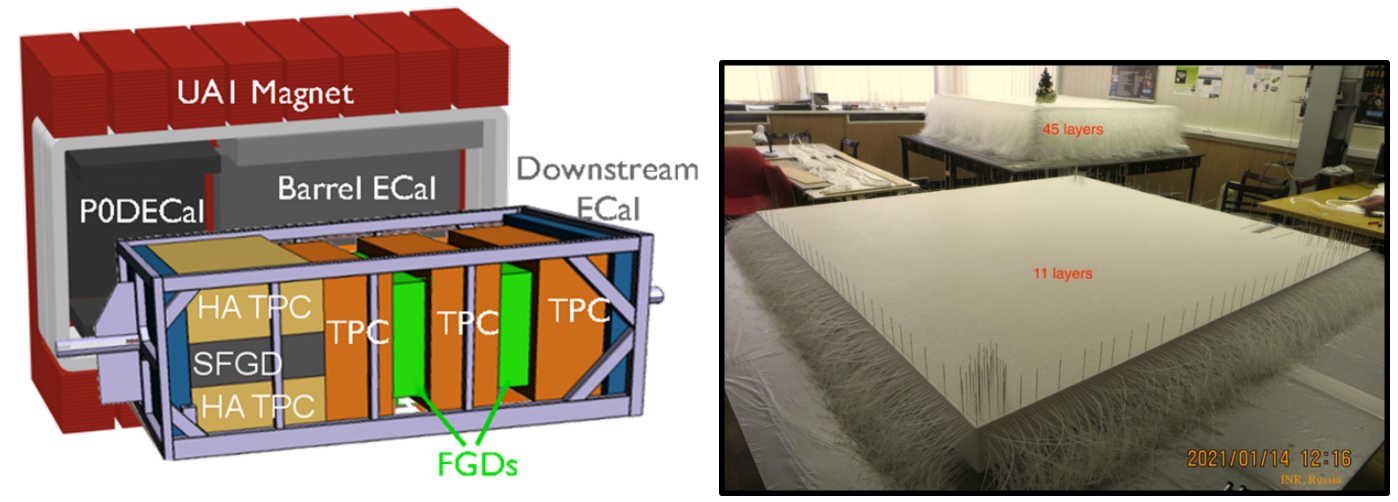

Figure 4: The left figure shows an exploded view of the upgraded ND280 detector (the new components are the HA TPC and SFGD in addition to the ToF pannels (not shown). Neutrinos enter from the left of the image. This figure adapted from Refs $[1,16]$. The right figure shows part of the assembly of $\sim 2$ million scintillator cubes used to build the SFGD at INR in Moscow.
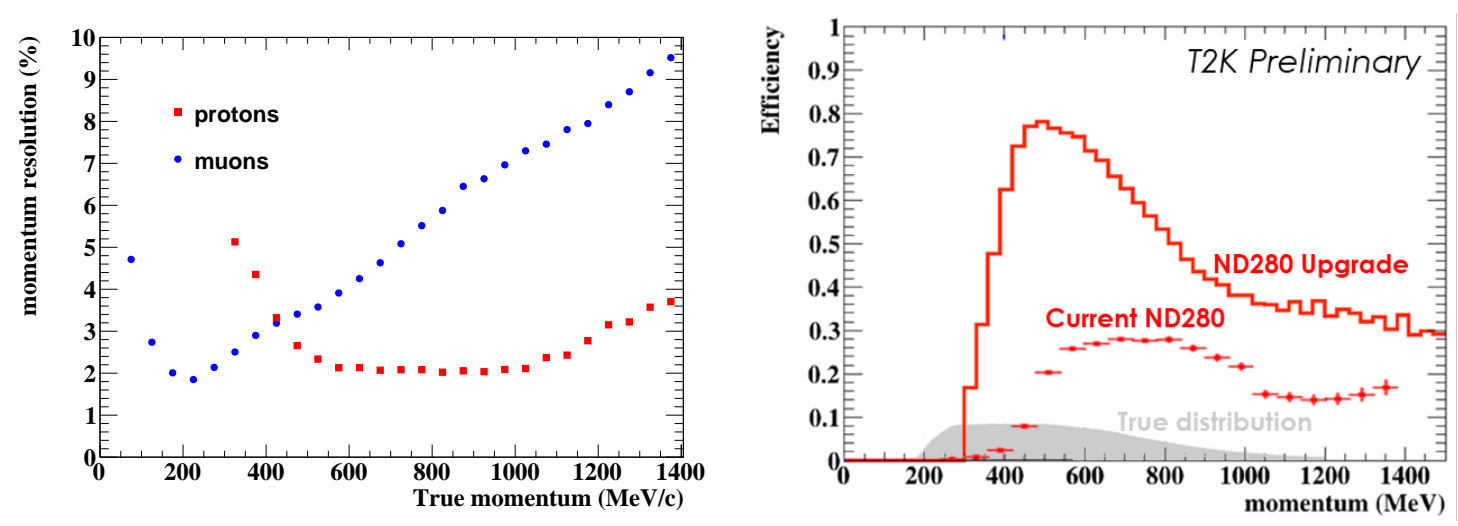

Figure 5: The left plot shows the upgraded ND280's expected resolution for muon and proton momenta all as a function of their respective momenta as reported in [21]. For reference, the typical momentum resolution of the current ND280 is $\sim 11 \%(\sim 9 \%)$ for muons (protons). The right plot shows the efficiency for selecting protons in a typical " $\mathrm{CC} 0 \pi$ " event selection at the current ND280 and using the upgrade. The predicted true distribution of proton momentum from the "NEUT" simulation is also shown.

\section{Summary}

$\mathrm{T} 2 \mathrm{~K}$ is making world-leading measurements of neutrino oscillations, having provided the first constraints on the $\delta_{C P}$ PMNS parameter to exclude CP-conserving values at more than $90 \%$ confidence level. Through increased statistics and detector upgrades, T2K will continue to improve its measurements in the run up to it's successor: the Hyper-Kamiokande experiment (due to start data taking in 2027) [22]. In particular, the expanded capabilities of the upgraded ND280 offer extensive opportunities for significant reduction of crucial systematic uncertainties associated with the incoming neutrino flux prediction and neutrino interaction modelling. 


\section{References}

[1] K. Abe et al. (T2K), Nucl. Inst. Meth. A 659, 106 (2011), arXiv:1106.1238 [phys.ins-det] .

[2] K. Abe et al. (T2K), Phys. Rev. D 103, 112008 (2021), arXiv:2101.03779 [hep-ex] .

[3] Z. Maki, M. Nakagawa, and S. Sakata, Prog. Theor. Phys. 28, 870 (1962).

[4] B. Pontecorvo, Sov. Phys. JETP 26, 984 (1968).

[5] K. Abe et al. (T2K), Phys. Rev. D 87, 012001 (2013).

[6] L. Alvarez-Ruso et al., Prog. Part. Nucl. Phys. 100, 1 (2018), arXiv:1706.03621 [hep-ph] .

[7] Y. Hayato and L. Pickering, The NEUT Neutrino Interaction Simulation, arXiv:2106.15809 [hep-ph] .

[8] P. Dunne, "Latest neutrino oscillation results from T2K," NEUTRINO 2020.

[9] K. Abe et al. (T2K), Nature 580, 339 (2020), [Erratum: Nature 583, E16 (2020)], arXiv:1910.03887 [hep-ex] .

[10] N. Abgrall et al. (NA61/SHINE), Eur. Phys. J. C 76, 617 (2016), arXiv:1603.06774 [hep-ex] .

[11] A. Himmel, "New oscillation results from the NOvA experiment," NEUTRINO 2020.

[12] M. G. Aartsen et al. (IceCube), Phys. Rev. Lett. 120, 071801 (2018), arXiv:1707.07081 [hep-ex] .

[13] Y. Nakajima, "Recent results and future prospects from Super-Kamiokande," NEUTRINO 2020.

[14] Y. Oyama, PoS NuFact2019, 054 (2020), arXiv:2004.06877 [physics.acc-ph] .

[15] C. Simpson et al. (Super-Kamiokande), Astrophys. J. 885, 133 (2019), arXiv:1908.07551 [astro-ph.HE] .

[16] K. Abe et al. (T2K), T2K ND280 Upgrade - Technical Design Report, arXiv:1901.03750 [physics.ins-det] .

[17] D. Attié et al., Nucl. Instrum. Meth. A 957, 163286 (2020), arXiv:1907.07060 [phys.ins-det] .

[18] A. Korzenev et al., A $4 \pi$ time-of-flight detector for the ND280/T2K upgrade, arXiv:2109.03078 [physics.ins-det] .

[19] A. Blondel et al., JINST 13, P02006 (2018), arXiv:1707.01785 [physics.ins-det] .

[20] L. Munteanu, S. Suvorov, S. Dolan, D. Sgalaberna, S. Bolognesi, S. Manly, G. Yang, C. Giganti, K. Iwamoto, and C. Jesús-Valls, Phys. Rev. D 101, 092003 (2020), arXiv:1912.01511 [physics.ins-det] .

[21] S. Dolan et al., Sensitivity of the Upgraded T2K Near Detector to constrain neutrino and anti-neutrino interactions with no mesons in the final state by exploiting nucleon-lepton correlations, arXiv:2108.11779 [hep-ex] .

[22] K. Abe et al. (Hyper-Kamiokande), (2018), arXiv:1805.04163 [physics.ins-det] . 